Preface

\title{
Maintaining Hemostasis and Preventing Thrombosis in Coronavirus Disease 2019 (COVID-19)-Part I
}

\author{
Emmanuel J. Favaloro, PhD, FFSc (RCPA) ${ }^{1}$ Giuseppe Lippi, MD ${ }^{2}$ \\ ${ }^{1}$ Department of Haematology, Institute of Clinical Pathology and \\ Medical Research (ICPMR), Sydney Centres for Thrombosis and \\ Haemostasis, Westmead Hospital, Westmead, Australia \\ 2 Section of Clinical Biochemistry, University of Verona, Verona, Italy \\ Semin Thromb Hemost 2020;46:757-762.
}

Welcome to another issue of Seminars in Thrombosis and Hemostasis (STH). This issue is published under the "banner" of "Maintaining Hemostasis and Preventing Thrombosis in Coronavirus Disease 2019 (COVID-19)," this being the first such issue (or "Part I"). COVID-19 is caused by severe acute respiratory syndrome coronavirus 2 (SARS-CoV-2). Believed to originate from Wuhan City in China, with the first reported case in December 2019, at the time of writing, there were over 26 million cases reported worldwide, and nearly 900,000 attributable deaths. ${ }^{1}$ From a smattering of reports in the scientific literature in late $2019(n=228)$, there is now over 51,000 publications ascribed to COVID-19 in PubMed, including over 5,000 reviews. Given this enormous explosion of information, it is virtually impossible to keep up with the literature on COVID-19. Also given this plethora of information, one wonders if there is much point adding yet more information into the pile? Once COVID-19 exploded into our collective minds, the editorial team of STH gave serious thought to this question. One situation that was clear to us was that given the great interest and initial thirst for knowledge, all journals became interested in publishing on COVID-19 and, indeed, some articles have been fast tracked to publication that have since been retracted, even by high impact factor journals. The STH board decided that, yes, STH needed to publish on COVID-19, but no, we did not want to publish just anything on COVID-19, and so we actually took a fairly resistant stance initially to acceptance of COVID-19 articles, just because they mentioned COVID-19. Primarily, STH did not want to publish misinformation, or additional "me too" articles (e.g., reviews similar to those that had already been published elsewhere). It was felt that we have a smart Editorial Board. The Board has kept abreast of the literature, even for COVID-19, despite that being a tough task. Hence, our initial approach to COVID-19, as noted in this issue of STH, was to canvass from the
STH Editorial Board, a series of commentaries around various aspects of COVID-19, although given the journal scope, obviously related primarily to thrombosis and hemostasis. The guest editors of this issue series also started the ball rolling for STH by preparing a commentary that we published some time ago, on laboratory tests that might be recommended for routine assessment of COVID-19 patients, ${ }^{2}$ and which we could then use as a kind of template to guide further commentaries. We wanted these contributions to reflect a range of topics, but be of high quality. We also needed to name the issue series. We knew early on, and this became then absolutely clear from postmortem studies that despite the disease manifesting initially as a respiratory disease, thrombosis is a hallmark of most (if not all) severe/critical COVID-19 cases. Hence, the issue name "Maintaining Hemostasis and Preventing Thrombosis in Coronavirus Disease 2019 (COVID-19)" was coined, which we hope will become a kind of mantra to us all.

- Fig. 1 gives a kind of overview of COVID-19 from our perspective.

The issue starts with themes around thrombosis, given this is a major feature in patients with severe COVID-19. The first contribution is from Di Minno et al, who provide an updated meta-analysis of literature studies around COVID19 and venous thromboembolism (VTE), ${ }^{3}$ thus providing the backdrop to a major theme of this series. COVID-19 presents with a wide spectrum of clinical presentations, leading in some cases to a critical care respiratory condition with poor long-term outcomes and residual chronic disability. A major concern in severe COVID-19 is represented by the concomitant prothrombotic state. However, contrasting data are available about the prevalence of VTE, including deep vein thrombosis (DVT) and/or pulmonary embolism (PE). The authors performed a detailed search on the association of COVID-19 with thromboembolic complications in the main
Address for correspondence Emmanuel J. Favaloro, PhD, FFSc (RCPA), Department of Haematology, Institute of Clinical Pathology and Medical Research (ICPMR), Westmead Hospital, Westmead 2145, Australia (e-mail: emmanuel.favaloro@ health.nsw.gov.au).
Issue Theme Maintaining Hemostasis and Preventing Thrombosis in COVID-19-Part I; Guest Editors: Emmanuel J. Favaloro, PhD, FFSc (RCPA), and Giuseppi Lippi, MD.

DOI https://doi.org/ 10.1055/s-0040-1717139. ISSN 0094-6176.
Copyright $\odot 2020$ by Thieme Medical Publishers, Inc., 333 Seventh Avenue, New York, NY 10001, USA. Tel: +1(212) 760-0888.

\section{License terms

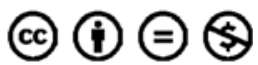




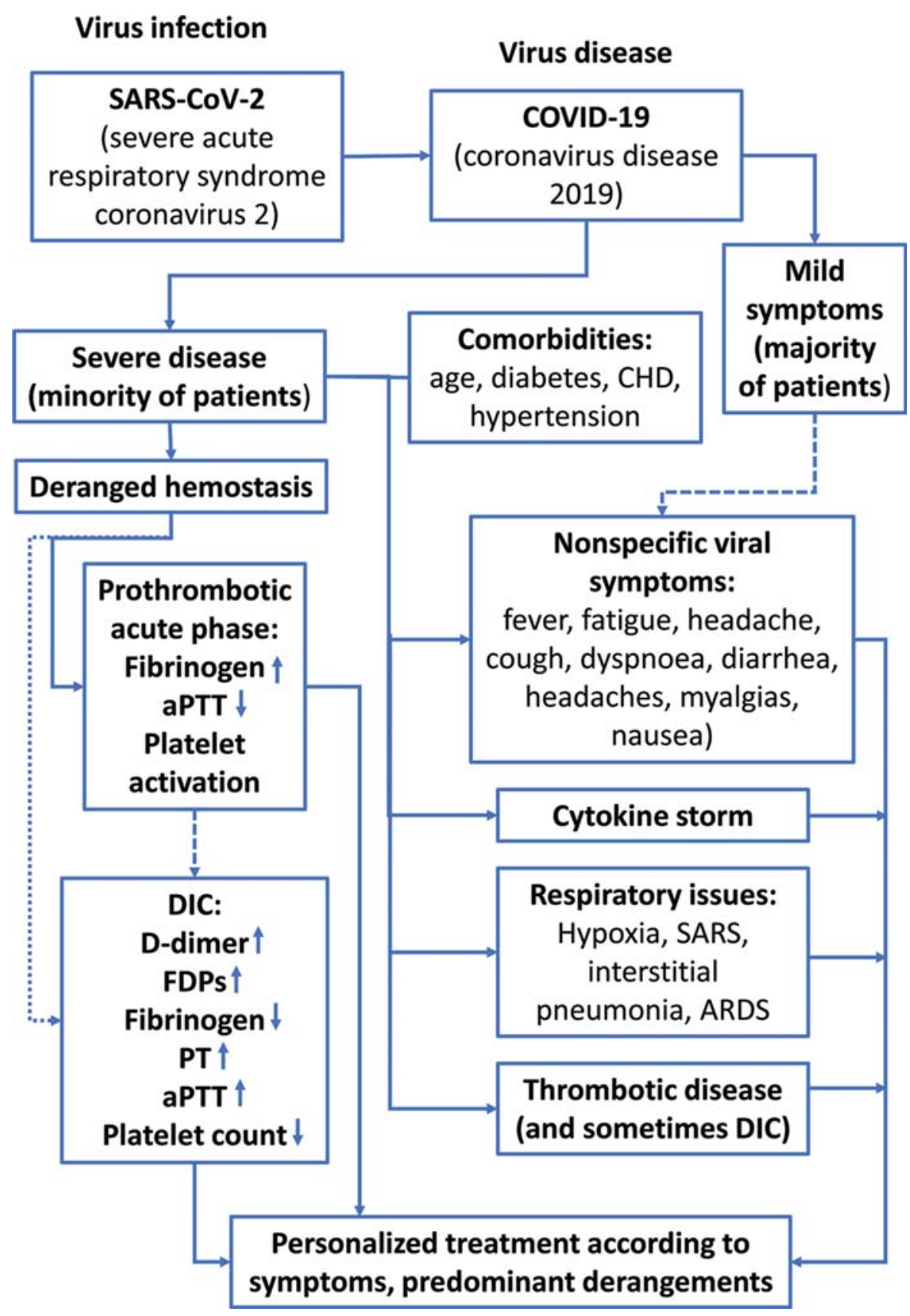

Fig. 1 A summary of COVID-19 issues. aPTT, activated partial thromboplastin time; ARDS, acute respiratory distress syndrome; CHD, coronary heart disease; DIC, disseminated intravascular coagulation; FDP, fibrin/fibrinogen degradation products; PT, prothrombin time; SARS, severe acute respiratory syndrome.

electronic databases (PubMed, Web of Science, Scopus) according to Preferred Reporting Items for Systematic Reviews and Meta-Analyses (PRISMA) guidelines. The weighted mean prevalence (WMP) with 95\% confidence intervals $(95 \% \mathrm{CI})$ was calculated with the random effects model. Twenty studies enrolling 1,988 COVID-19 subjects were included. The WMP of VTE was 31.3\% (95\% CI, 24.3$39.2 \%$ ). The WMP of DVT was $19.8 \%$ (95\% CI, 10.5-34.4\%), whereas the WMP of PE was $18.9 \%$ (95\% CI, 14.4-24.3\%). Similar results were obtained when specifically analyzing studies on patients admitted to intensive care units (ICU) and on patients under antithrombotic prophylaxis. Regression models showed that an increasing age was associated with a higher prevalence of VTE ( $Z$-value: $3.11, p=0.001)$, DVT $(Z$ value: $2.33, p=0.002)$, and PE ( $Z$-value: $3.03, p=0.002)$, while an increasing body mass index (BMI) was associated with enhanced prevalence of PE ( $Z$-value: $2.01, p=0.04)$. Male sex did not impact the evaluated outcomes.

The second contribution, from Sam Schulman, ${ }^{4}$ is a commentary on "COVID-19, prothrombotic factors, and VTE." Dr. Schulman identifies that COVID-19 causes several changes in the prothrombotic direction and this can be explained by the profound inflammatory response as well as hypoxia. Many of the changes seen are similar to what is otherwise observed in sepsis, although the hypoxia seen in COVID-19 pneumonia is likely an additional aggravating factor. This may result in 
increased incidence of in situ pulmonary thrombosis, as potentially distinct from $\mathrm{PE}$, but which may similarly have been the case in critically ill patients with influenza A H1N1. VTE, despite chemoprophylaxis in patients in ICU, is not a new phenomenon and it is not clear whether this occurs more often with COVID-19.

Continuing the thrombosis theme, another commentary from Thachil and Srivastava asks: "Is SARS-2 corona virus associated with hemostatic lung abnormality in COVID-19 pulmonary thrombosis or pulmonary embolism?"5 They term the COVID-19-associated hemostasis abnormalities as "CAHA," and as representing a localized thrombotic phenomenon, which thus requires specific assessment and appropriate therapeutic response compared with VTE. They advise health care providers to be cognizant of the significant possibility of pulmonary microthrombi in these patients and should keep a low threshold for investigating this complication in all patients. This is important because pulmonary thrombosis and PE have different pathogenesis and may hence need different treatments. In the case of pulmonary microthrombi, this may be a combination of other treatments in addition to anticoagulants. The authors remain positive, and are sure that we will "win in this battle against an unseen but now well-known virus," but we should aim to learn a large amount quickly, regarding both COVID19 pathology and the potential therapies to limit short-term and long-term morbidity and mortality in infected patients.

A fourth installment, another commentary, is provided by Levi and Thachil, who pose a slightly different question: "Is COVID-19 coagulopathy a reflection of disseminated intravascular coagulation (DIC) and/or thrombotic microangiopathy?" ${ }^{6}$ The authors reflect that severe COVID-19 infection is associated with coagulation abnormalities that encompass elements reminiscent of both DIC and thrombotic microangiopathy. However, this coronavirus infection seems to cause a specific coagulopathy that is more localized and distinctly different from "classical" DIC or thrombotic microangiopathy syndromes. The specific coagulopathic features of this disease are likely relevant in view of the high incidence of thromboembolic complications in severely affected patients and may present an important point of impact for (supportive) preventive or therapeutic management strategies to improve the clinical outcome.

Next, Horowitz and Brenner continue the discussion around thrombosis in COVID-19 in another commentary, but this one focusing on cancer. ${ }^{7}$ The authors begin by discussing that VTE is common in cancer patients, increased five- to sevenfold compared with those without cancer, while fatal PE is three times more common in the cancer population relative to noncancer patients. The authors then discuss the mechanisms of thrombosis in cancer and COVID19 patients, as well as clinical and therapeutic management. They conclude that extrapolating the standard of care of antithrombotic strategy to cancer patients with COVID-19 is challenging due to paucity of data and high risk for both thrombosis and bleeding. They also believe that rapidly emerging data will hopefully foster management regimens for these critically ill patients.
The issue continues on the thrombosis in COVID-19 theme, but switches to the topic of "therapy" in the next few installments. First, Hashemi and colleagues provide a commentary on pharmacotherapy for the prevention and management of thrombosis in COVID-19. ${ }^{8}$ As highlighted earlier, the existing body of evidence, consisting of pathophysiological, epidemiologic, and postmortem studies, indicates the frequent presence of a prothrombotic state in COVID-19, and suggests there may be a close association between inflammatory and thrombotic pathways. The authors believe that antithrombotic therapy for known (or incident) thrombotic disease in patients with COVID-19 will be largely similar to pre-COVID-19 era, with specific attention to the risk of pulmonary hemorrhages, acute deterioration of hepatic and renal function, need for invasive procedures, and drug-drug interactions with investigational COVID-19 therapies. However, optimal prevention of thrombotic events in this prothrombotic condition faces multiple unknowns with regard to right dose and regimen. Additional epidemiological and comparative effectiveness studies will help identify the highest risk subgroups, and optimal preventive strategies to safely mitigate the risk of thrombotic events. This commentary in part summarizes findings reported by them and others in earlier larger reviews. ${ }^{9,10}$

Next come two articles on passive immunity for COVID19 , as reflecting one option for managing the severity of COVID-19. First, Lindholm and colleagues provide a review on therapeutic aspects, including the use of "convalescent" plasma. ${ }^{11}$ In the ongoing pandemic of COVID-19, the novel virus SARS-CoV-2 is infecting a naive population. The innate immunity of infected patient is almost unable to mount an effective defense, resulting in a severe illness with substantial morbidity and mortality. As most treatment modalities including antivirals and anti-inflammatory agents may be ineffective, an immunologic approach is needed. The mechanism of innate immunity to this viral illness is not fully understood. Passive immunity becomes an important avenue for managing these patients. In this article, the immune responses of COVID-19 patients are appraised. As SARS-CoV2 has many characteristics in common with two other viruses, SARS-CoV that caused severe acute respiratory syndrome (SARS) in 2002-2003 and MERS-CoV that caused the Middle East respiratory syndrome (MERS) in 2012, the experiences learned from the use of passive immunity in treatment can be applied to COVID-19. The immune response includes the appearance of immunoglobulin $\mathrm{M}$, almost contemporary to that of immunoglobulin $\mathrm{G}$, along with neutralizing antibodies. Convalescent plasma obtained from patients recovered from the illness with high titers of neutralizing antibodies was successful in treating many COVID19 patients. The factors that determine responses compared with those seen in SARS and MERS are also reviewed. As there are yet no approved vaccines against all these three viruses, it remains a challenge in the ongoing development of an effective vaccine for COVID-19.

A commentary on the challenges in the production of convalescent hyperimmune plasma in the age of COVID-19 is then provided by Franchini and colleagues. ${ }^{12}$ First, no 
uniformly standardized therapy has been endorsed for COVID-19 and several drugs are currently being investigated in several nonrandomized or randomized trials for patients with life-threatening COVID-19.9,10 These agents include steroids, chloroquine, antiviral medications (i.e., remdesivir, lopinavir/ritonavir), and anti-inflammatory agents (i.e., tocilizumab, sarilumab, baricitinib), the last ones being utilized following the discovery of the involvement of SARS-CoV-2induced inflammatory response in the pathogenesis of SARS. However, experiences from previous coronavirus epidemics (SARS and MERS) indicate that convalescent plasma collected from recovered COVID-19 patients, containing neutralizing antibodies against SARS-CoV-2, could be a potentially effective therapeutic weapon. However, there are many technical and regulatory hurdles to overcome before effective therapies can be assured. The authors describe a multicenter interventional single-arm trial initiated in their country (Italy) to produce hyperimmune plasma for treating critical patients with COVID-19.

A different type of ("preventative") therapy for COVID-19 is explored by Zadow and colleagues, ${ }^{13}$ who discuss COVID-19, coagulopathy, and exercise, focusing on interactions that may influence health outcomes. The proinflammatory cytokine storm associated with COVID-19 negatively affects the hematological system, leading to coagulation activation and endothelial dysfunction, thus increasing the risk of both venous and arterial thrombosis. ${ }^{14}$ Coagulopathy has been reported as associated with mortality in people with COVID-19 and is partially reflected by enhanced D-dimer levels. ${ }^{15-17}$ Poor vascular health, which is associated with cardiometabolic health conditions frequently reported in people with severer forms of COVID-19, might exacerbate the risk of coagulopathy and mortality. Sedentary lifestyles might also contribute to the development of coagulopathy, and physical activity participation has been inherently lowered due to lockdowns and athome regulations, established to slow down the spread of this highly infectious disease. It is possible that COVID-19, coagulation, and reduced physical activity may contribute to generate a "perfect storm," where each fuels the other and potentially increases mortality risk. Several pharmaceutical agents are being explored to treat COVID-19, ${ }^{9,10}$ but potential negative consequences are associated with their use. Exercise is known to mitigate many of the identified side-effects from the pharmaceutical agents being trialed, but has not yet been considered as part of COVID-19 management. From the limited available evidence in people with cardiometabolic health conditions, low- to moderate-intensity exercise might have the potential to positively influence biochemical markers of coagulopathy, while high-intensity exercise is likely to increase thrombotic risk. Therefore, low- to moderate-intensity exercise could be an adjuvant therapy for people with mild to moderate COVID-19 and reduce the risk of developing severe symptoms of illness which are associated with enhanced mortality.

This issue of STH then turns away from the prothrombotic side of COVID-19 to explore the less well investigated "bleeding side" of COVID-19. Dr. Dorgalaleh starts the discussion with a commentary on bleeding and bleeding risk in
COVID-19, ${ }^{18}$ advising that although hypercoagulability and thrombotic events are common in COVID-19, bleeding may occur at any time during the course of disease. Several factors make patients with COVID-19 prone to bleeding, including thrombocytopenia, hyperfibrinolytic state, consumption of coagulation factors, and thromboprophylactic administration of anticoagulants. Also, the proposed cytokine storm, prolonged tissue hypoxia, and direct invasion of affected tissues may reflect other possible causes of bleeding. Although thrombosis is relatively well studied in COVID-19, bleeding and bleeding risk appear to be the forgotten side of this story, most probably due to the less-fatal consequences. However, hemorrhagic diatheses represent a significant morbidity and potential cause of death in COVID-19, in at least a subset of patients. The author concludes that due to high rate of coagulopathy among COVID-19 patients, the risk of bleeding should always be considered in every case, as bleeding, although rare, may be one of the first clinical presentations at the time of diagnosis.

Continuing this theme, at least in principle, is the next contribution from Coppola and colleagues, who provide another commentary, this time on the topic of confronting COVID-19 in patients with hemophilia and congenital bleeding disorders (CBDs). ${ }^{19}$ The unexpectedly large numbers of severely affected COVID-19 patients have overwhelmed health care systems worldwide, with most human and economic resources devoted to critical epidemic emergency. Moreover, the need for mobility restrictions is limiting access to usual standards of medical care, which is likely harmful for patients with chronic diseases and/or requiring regular clinical followup. This is the case for CBD, especially the most symptomatic forms, such as severe hemophilia. Many issues are being faced by these patients in the COVID-19 era. Hemophilia treatment centers and the hemophilia community are currently defining safe modalities for facing the COVID-19 pandemic. Although clinical data in CBD patients with COVID-19 are lacking, they should undergo all treatment modalities available, including invasive approaches, provided that their bleeding risk is covered by adequate hemostatic prophylaxis. As our knowledge and clinical experience develop, we will learn whether the coagulation defects present in people with hemophilia and CBD may influence the epidemiologic impact and severity of COVID-19.

We now move the issue to a series of miscellaneous articles that continue on the COVID-19 story. First, a commentary from Larsen and colleagues around the potential role of platelets in the pathology of COVID-19, including the link between thrombocytopenia and disease severity, and considerations on the potential role for platelet function and/or platelet activation testing in COVID-19 patients. ${ }^{20}$ First, although it appears that mild thrombocytopenia is a common finding in COVID-19 patients, a platelet count $<100 \times 10^{9} / \mathrm{L}$ seems rare and should be interpreted as an indicator of the presence or development of coagulopathy. This could also help identify patients who could benefit from thromboprophylaxis. Also, platelet activation in the pulmonary microcirculation could not only contribute to the procoagulant activity described in COVID-19 patients, but may also aggravate damage to the lung 
parenchyma and contribute to the respiratory distress and frequent need for mechanical ventilation, which is a hallmark of severe COVID-19.

Parra-Izquierdo and Aslan take this discussion on platelets further in the next commentary, discussing platelet heterogeneity and host immune response in COVID-19. ${ }^{21}$ As thrombocytopenia, systemic microvascular thrombosis, and elevated D-dimer levels reflect COVID-19 severity, cellular effectors of hemostasis and thrombosis-especially platelets -likely participate in COVID-19 pathogenesis. However, specific roles for platelets in COVID-19 as disease drivers, biomarkers, and therapeutic targets remain unspecified. Here, the authors highlight how platelets may be affected by COVID-19 in a manner supporting pathology-and, which offers insights into COVID-19 susceptibility, progression, and resolution.

Thachil and Lisman then investigate the role of pulmonary megakaryocytes in COVID-19, potentially in thrombosis and fibrosis. $^{22}$ Autopsy reports of COVID-19 patients who succumbed to their viral infection have been published despite concerns about health care professional's safety. One of the unusual findings in COVID-19 lung autopsy reports is the increase in pulmonary megakaryocytes. Although the presence of megakaryocytes in the lungs is a well-established concept in the medical literature, it is still not widely accepted in the clinical fraternity. In this article, the authors discuss the role of lung megakaryocytes in relation to clinicopathological findings in COVID-19 and how this may impact on our understanding of ARDS, pulmonary thrombi, and lung fibrosis, in general.

de Maat et al then provide a commentary on the impaired breakdown of bradykinin and its metabolites as a possible cause for pulmonary edema in COVID-19 infection. ${ }^{23}$ The authors describe the pathophysiological relationships as well as potential implications on therapeutic strategies in COVID-19.

Vadasz and colleagues then provide a commentary on immune-mediated coagulopathy in COVID-19 infection. ${ }^{24} \mathrm{~A}$ variety of viruses are frequently blamed as a trigger for immune-mediated inflammation and autoimmunity. Several underlying mechanisms have been suggested, including virus' direct overstimulatory effect on both innate and adaptive immune responses, cross-reactivity with self-antigens, and, lastly, direct effect on regulatory functions. However, the high infectivity and damaging effect of COVID-19 on respiratory tract, endothelial cells, as well as on both innate and adaptive immunity seem to be far beyond those of many other viruses. The authors discuss the "cytokine storm" associated with COVID-19, and damage to endothelial cells and cardiovascular involvement, as key to the pathophysiology of COVID-19.

Next, Dr. Kwaan explores the role of the fibrinolytic system in COVID-19, reflecting on the journey from transmission to organ injury and sequelae. ${ }^{25}$ Many aspects of COVID-19 are similar to those seen in SARS and MERS, including angiotensin-converting enzyme 2 as receptor for this virus. ${ }^{14}$ Impaired fibrinolysis was observed in post-SARS complications. Impaired fibrinolysis is present in pneumonia and acute lung injuries; accordingly, this commentary is devoted to reviewing evidence for possible involvement of fibrinolytic system in transmission, pulmonary complications, and sequelae of COVID-19. Several possible drug targets that alter the activity of components of the fibrinolytic system are also discussed.

The last full-length article in this issue of $S T H$ is a review of hematology laboratory abnormalities in patients with COVID-19 from Christensen and colleagues. ${ }^{26}$ While many infected individuals do not experience signs or symptoms, or experience only mild symptoms, a subset of individuals experience severe disease, which is often fatal. Several laboratory tests have been found abnormal in hospitalized patients, and some studies suggest some of these tests can predict an unfavorable outcome. These include markers of acute phase reaction (elevated C-reactive protein, erythrocyte sedimentation rate, white blood cell count, fibrinogen, procalcitonin, factor VIII, von Willebrand factor), signs of tissue injury (elevated lactic dehydrogenase, alanine aminotransferase, cardiac troponins), changes in hemostasis and coagulation (elevated D-dimer, prolonged prothrombin time, decreased platelets, decreased antithrombin, elevated factor VIII, and von Willebrand factor), and decreased lymphocyte count. Additional studies are needed to confirm the most ideal panel of tests, and confirm the efficiency of laboratory tests to predict clinical outcome, as well as ideal anticoagulation management. This contribution is, in large part, an update and extension of the first COVID-19 article published in $S T H,{ }^{2}$ and thus represents a kind of closed circle.

This issue of the journal concludes with a series of Letters to the Editor (correspondence) around COVID-19. First, Padley and colleagues describe a case of catheter-directed thrombolysis in a patient with severe COVID-19 pneumonia on extracorporeal membrane oxygenation. ${ }^{27}$ Next Dorgalaleh and colleagues present a case series of patients with CBD, who appear to be less severely affected by SARS-CoV-2 compared with non-CBD patients, thus raising a question of inherited hypocoagulability overcoming, at least in part, the acquired hypercoagulability of COVID-19. ${ }^{28}$ Then, Mattioli et al describe a case of atypical spontaneous hematomas in a patient with severe COVID-19. ${ }^{29}$ The final installment in this first issue of COVID-19 is by Henry et al, who identify changes in the circulating plasminogen concentration at admission in patients with COVID-19. ${ }^{30}$ In brief, they observed that patients with COVID-19 present to the emergency department with normal levels of plasminogen, but those progressing to severe disease develop mildly lower values, which may be suggestive of consumption for fibrinolysis activation. They recommend that future studies should be aimed at investigating the pathophysiology of COVID-19associated coagulopathy, including serial measurements of multiple components of the fibrinolytic system.

We thank all the authors to this inaugural issue for their original and comprehensive contributions, and we hope the readers of STH enjoy this first installment in this series. Although in pages, the issue represents a standard issue length, the sheer number of contributions $(n=23)$ reflects a wide range of topics and viewpoints which the readers of STH should appreciate. 
Conflict of Interest

None declared.

\section{References}

1 COVID-19 Dashboard by the Center for Systems Science and Engineering (CSSE) at Johns Hopkins University (JHU). Available at: https://www.arcgis.com/apps/opsdashboard/index.html\#/ bda7594740fd40299423467b48e9ecf6. Accessed September 4, 2020

2 Favaloro EJ, Lippi G. Recommendations for minimal laboratory testing panels in patients with COVID-19: potential for prognostic monitoring. Semin Thromb Hemost 2020;46(03):379-382

3 Di Minno A, Ambrosino P, Calcaterra I, Di Minno MND. COVID-19 and venous thromboembolism: a meta-analysis of literature studies. Semin Thromb Hemost 2020;46(07):763-771

4 Schulman S. COVID-19, prothrombotic factors and venous thromboembolism. Semin Thromb Hemost 2020;46(07):772-776

5 Thachil J, Srivastava A. SARS-2 corona virus associated hemostatic lung abnormality in COVID-19: is it pulmonary thrombosis or pulmonary embolism? Semin Thromb Hemost 2020;46(07): 777-780

6 Levi M, Thachil J. COVID-19 coagulopathy: disseminated intravascular coagulation and thrombotic microangiopathy: either, neither or both. Semin Thromb Hemost 2020;46(07):781-784

7 Horowitz NA, Brenner B. Thrombosis and hemostasis issues in cancer patients with COVID-19. Semin Thromb Hemost 2020;46 (07):785-788

8 Hashemi A, Madhavan MV, Bikdeli B. Pharmacotherapy for prevention and management of thrombosis in COVID-19. Semin Thromb Hemost 2020;46(07):789-795

9 Bikdeli B, Madhavan MV, Jimenez D, et al; Global COVID-19 Thrombosis Collaborative Group, Endorsed by the ISTH, NATF, ESVM, and the IUA, Supported by the ESC Working Group on Pulmonary Circulation and Right Ventricular Function. COVID-19 and Thrombotic or thromboembolic disease: implications for prevention, antithrombotic therapy, and follow-up: JACC stateof-the-art review. J Am Coll Cardiol 2020;75(23):2950-2973

10 Bikdeli B, Madhavan MV, Gupta A, et al; Global COVID-19 Thrombosis Collaborative Group. Pharmacological agents targeting thromboinflammation in COVID-19: review and implications for future research. Thromb Haemost 2020;120(07):1004-1024

11 Lindholm PF, Ramsey G, Kwaan HC. Passive immunity for COVID 19-a commentary on therapeutic aspects including convalescent plasma. Semin Thromb Hemost 2020;46(07):796-803

12 Franchini F, Del Fante C, Klersy C, Glingani C, Percivalle E, Baldanti F, Perotti C. Challenges in the production of convalescent hyperimmune plasma in the age of COVID-19. Semin Thromb Hemost 2020;46(07):804-806

13 Zadow EK, Wundersitz DWT, Hughes DL, et al. COVID-19, coagulopathy and exercise: Interactions that may influence health outcomes. Semin Thromb Hemost 2020;46(07):807-814

14 Henry BM, Vikse J, Benoit S, Favaloro EJ, Lippi G. Hyperinflammation and derangement of renin-angiotensin-aldosterone system in COVID-19: a novel hypothesis for clinically suspected hypercoagulopathy and microvascular immunothrombosis. Clin Chim Acta 2020;507:167-173

15 Lippi G, Favaloro EJ. D-dimer is associated with severity of coronavirus disease 2019: a pooled analysis. Thromb Haemost 2020;120(05):876-878

16 Favaloro EJ, Thachil J. Reporting of D-dimer data in COVID-19: some confusion and potential for misinformation. Clin Chem Lab Med 2020;58(08):1191-1199

17 Thachil J, Longstaff C, Favaloro EJ, Lippi G, Urano T, Kim PYSSC Subcommittee on Fibrinolysis of the International Society on Thrombosis and Haemostasis. The need for accurate D-dimer reporting in COVID-19: communication from the ISTH SSC on fibrinolysis. J Thromb Haemost 2020;18(09):2408-2411

18 Dorgalaleh A. Bleeding and bleeding risk in COVID-19. Semin Thromb Hemost 2020;46(07):815-818

19 Coppola A, Tagliaferri A, Rivolta GF, Quintavalle G, Franchini M. Confronting COVID-19: issues in hemophilia and congenital bleeding disorders. Semin Thromb Hemost 2020;46(07):819-822

20 Larsen JB, Pasalic L, Hvas A-M. Platelets in coronavirus disease 2019 (COVID-19). Semin Thromb Hemost 2020;46(07):823-825

21 Parra-Izquierdo I, Aslan JE. Perspectives on platelet heterogeneity and host immune response in COVID-19. Semin Thromb Hemost 2020;46(07):826-830

22 Thachil J, Lisman T. Pulmonary megakaryocytes in COVID-19roles in thrombi and fibrosis. Semin Thromb Hemost 2020;46 (07):831-834

23 de Maat S, de Mast Q, Danser AHJ, van de Veerdonk PL, Maas C. Impaired breakdown of bradykinin and its metabolites as a possible cause for pulmonary edema in COVID-19 infection. Semin Thromb Hemost 2020;46(07):835-837

24 Vadasz Z, Brenner B, Toubi E. Immune-mediated coagulopathy in COVID-19 infection. Semin Thromb Hemost 2020;46(07): $838-840$

25 Kwaan HC. COVID-19: the role of fibrinolytic system from transmission to organ injury and sequelae. Semin Thromb Hemost 2020;46(07):841-844

26 Christensen B, Favaloro EJ, Lippi G, Van Cott EM. Hematology laboratory abnormalities in patients with coronavirus disease 2019 (COVID-19). Semin Thromb Hemost 2020;46(07):845-849

27 Padley GJ, Desai SS, Weaver C, Price LC, Arachchillage DJ, Ridge CA. Catheter-directed thrombolysis in a patient with severe COVID19 pneumonia on extracorporeal membrane oxygenation. Semin Thromb Hemost 2020;46(07):850-852

28 Dorgalaleh A, Dabbagh A, Tabibian S, et al. Patients with congenital bleeding disorders appear to be less severely affected by SARSCoV-2 - inherited hypocoagulability overcoming acquired hypercoagulability of COVID-19? Semin Thromb Hemost 2020;46(07): 853-855

29 Mattioli M, Benfaremo D, Fustini E, Gennarini S. Atypical spontaneous hematomas in a patient with severe COVID-19. Semin Thromb Hemost 2020;46(07):856-858

30 Henry BM, Benoit S, Hoehn J, Lippi G, Favaloro EJ, Benoit JL. Circulating plasminogen concentration at admission in patients with coronavirus disease 2019 (COVID-19). Semin Thromb Hemost 2020;46(07):859-862 